[Original article]

\title{
Psychologic distress and QOL in medical staff after a disaster : A longitudinal 4-year study
}

\author{
Shoji Yabuki ${ }^{122)}$, Kozue Takatsuki ${ }^{2)}$, and Kazuo Ouchi ${ }^{1)}$ \\ ${ }^{1)}$ Rehabilitation Center, Fukushima Medical University Hospital, Fukushima, Japan, ${ }^{2)}$ Department of \\ Pain Medicine, Fukushima Medical University School of Medicine, Fukushima, Japan
}

(Received August 3, 2021, accepted January 13, 2022)

\begin{abstract}
Aiming to improve post-disaster care of medical staff, we conducted an early and ongoing assessment of post-disaster psychologic distress and quality of life (QOL) in one center of a disaster-response hospital. Twelve days after the Great East Japan Earthquake, as the Fukushima Daiichi Nuclear Power Plant crisis was unfolding, we began a survey to examine the physical and mental state of medical staff to assess their motivation toward work. Surveys were administered in March 2011 (Survey 1), March 2012 (Survey 2), March 2013 (Survey 3), March 2014 (Survey 4), and March 2015 (Survey 5). Participants completed the Beck Depression Inventory (BDI), State-Trait Anxiety Inventory (STAI), EuroQol (EQ-5D), and MOS Short-Form 36-item Health Survey (SF-36). Although BDI scores significantly improved over time following Survey 1, participants in their 30s had significantly higher Survey 2 scores than those in their 40s/50s, and significantly higher Survey 3 scores than those in their 20s. STAI scores significantly improved over time following Survey 1. However, participants in their 30s had significantly higher Survey 3 scores than those in their 20s. EQ-5D scores did not significantly vary among survey time points or age groups. SF-36 physical functioning, role physical, social functioning, role emotional, and mental health subscale scores significantly improved over time. In conclusion, post-disaster longitudinal changes, including recovery period, differed among age groups. Thus, age should be taken into account in longitudinal evaluations of psychologic distress and QOL in medical staff after a disaster and, as more recent events suggest, during a pandemic.
\end{abstract}

Key words : Fukushima Daiichi Nuclear Power Plant accident, psychologic distress, QOL, disaster, longitudinal study

\section{Introduction}

On 11 March 2020, exactly 9 years after the Great East Japan Earthquake, the World Health Organization announced that the spread of COVID-19 (SARS-CoV-2 infection) had become a pandemic. This pandemic, in turn, has created a public health disaster that has increased psychologic distress, including anxiety and depression, in the global population ${ }^{1,2)}$, including medical staff ${ }^{3-5)}$. Understanding psychologic distress and changes in quality of life (QOL) among different age groups and professions, including medical staff during and after a disaster, may lead to better systems for addressing personal consequences of disaster response and post-disaster life ${ }^{6}$.

The Great East Japan Earthquake of 11 March 2011 initiated a complex disaster including a major tsunami, multiple aftershocks, and an ongoing nuclear power plant crisis. Psychologic distress was subsequently observed in many Japanese people, as nearly 20,000 were lost in the tsunami, and damage

Corresponding author: Shoji Yabuki MD, DMSc E-mail : yabuki@fmu.ac.jp

(C)2022 The Fukushima Society of Medical Science. This article is licensed under a Creative Commons [Attribution-NonCommercial-ShareAlike 4.0 International] license.

https://creativecommons.org/licenses/by-nc-sa/4.0/ 
at the Fukushima Daiichi Nuclear Power Plant resulted in substantial releases of radioactive materi$\mathrm{al}^{7-9)}$. Even though medical staff in Fukushima generally had more knowledge about radiation than the population at large, they, too, experienced disasterrelated anxiety. In the decade that followed, it has been possible to examine longitudinal changes in psychologic distress and quality of life $(\mathrm{QOL})^{10-13)}$. The purpose of this study was to assess longitudinal changes in psychologic distress and QOL in medical staff working in Fukushima Prefecture after the Great East Japan Earthquake, associated tsunami, and the Fukushima Daiichi Nuclear Power Plant accident, with the goal of generating data on how best to follow-up with medical staff experiencing psychologic distress and decreased QOL after a disaster. Although the 2011 disaster and the 2020 COVID-19 pandemic have different characteristics, they share various individual and societal risk issues, risk trade-offs, and measures to recognize and adapt to these risks ${ }^{14)}$. The present findings may provide useful information on how to follow-up with medical staff experiencing psychologic distress and decreased QOL during or after a pandemic.

\section{Subjects and methods}

We conducted a survey to examine the physical and mental state of medical staff after the disaster to assess their motivation toward work. We explained the purpose of the survey to staff and obtained their verbal consent to participate in this minimal risk research. The study received approval number 2020194 from the Ethics Committee of Fukushima Medical University, which is guided by local policy, national law, and the World Medical Association Declaration of Helsinki.

Participants were 19 medical staff working in the Rehabilitation Center at Fukushima Medical University Hospital, located about $60 \mathrm{~km}$ from the Fukushima Daiichi Nuclear Power Plant. Already equipped to deal with acute disease and trauma, this hospital was among those in Fukushima Prefecture specially designated for disaster response. Although the hospital itself was not severely damaged, its municipal water supply was interrupted, necessitating extreme conservation measures during the first week of disaster response. For close to a month, routine outpatient care was suspended, and patients with chronic conditions were evacuated to facilities outside the disaster area. Emergency care patients were received, treated, and, if necessary, transferred, until routine hospital practice could be resumed.

Radiation levels around the hospital were monitored and disclosed to all staff. A brief peak, on par with background radiation levels experienced in a commercial jet at cruising altitude, was followed by rapid exponential decay. Nevertheless, medical staff were worried about exposure to continuous low-dose radiation.

Participants were 10 men and 9 women, $36.9 \pm$ 12.3 years old (mean \pm standard deviation [SD]). Five participants were in their 20 s, eight were in their 30s, three were in their 40s, and three were in their 50s. Ten participants were physical therapists, three were occupational therapists, two were speech therapists, two were doctors, one was a nurse, and one was a nursing assistant. All participants had been working at the Rehabilitation Center when the Great East Japan Earthquake and the Fukushima Daiichi Nuclear Power Plant accident occurred.

This prospective, observational study began 12 days after the disaster, and continued for 4 years, with five surveys in total. The surveys were administered in March 2011 (Survey 1), March 2012 (Survey 2), March 2013 (Survey 3), March 2014 (Survey 4), and March 2015 (Survey 5).

The survey questionnaires included the Beck Depression Inventory (BDI), State-Trait Anxiety Inventory (STAI), EuroQol (EQ-5D), and MOS ShortForm 36-item Health Survey (SF-36). The informed consent process was conducted verbally, with all center staff agreeing to participate.

\section{$B D I$}

This self-report questionnaire measures the presence and severity of depression symptoms ${ }^{15,16)}$. Higher scores indicate greater depression : minimal depression $=0-9$, mild depression $=$ $10-16$, moderate depression $=17-29$, and severe depression $=30-63$. License to use the questionnaire is available from the following companies in Japan: SACCESS BELL (http://www.saccess55. co.jp/), Chiba Test Center (http://www.chibatc.co.jp), and Nihon Bunka Kagakusha (http://www.nichibun. co.jp/).

\section{STAI}

This self-report questionnaire measures the presence and severity of current symptoms of anxiety and generalized propensity to be anxious $^{17-19)}$. There are two subscales : the State Anxiety Scale (STAI-S) evaluates the current state of anxiety, and the Trait Anxiety Scale (STAI-T) evalu- 
ates relatively stable aspects of anxiety proneness. The score range for each subtest is 2080 ; higher scores indicate greater anxiety. A cutoff point of 39-40 for the STAI-S has been suggested to detect clinically significant symptoms. License to use the questionnaire is available from the following companies in Japan : SACCESS BELL (http:// www.saccess55.co.jp/), Chiba Test Center (http:// www.chibatc.co.jp), and Nihon Bunka Kagakusha (http://www.nichibun.co.jp/).

\section{$E Q-5 D$}

This questionnaire is a standardized non-disease-specific instrument for describing and evaluating health-related $\mathrm{QOL}^{20-22)}$. This instrument has five dimensions (mobility, self-care, usual activities, pain/discomfort, and anxiety/depression) with three levels each. The descriptive system defines $\left(3^{5}\right)=$ 243 different states with utility scores that range from 0.000-1.000. Higher scores indicate better QOL. The questionnaire was used after registration with the EuroQol Office (https://euroqol.org).

SF-36

This questionnaire is a multi-item generic health-related QOL survey intended to measure "general health concepts not specific to any age, disease, or treatment group"23-25). The SF-36 measures eight health domains : physical functioning $(\mathrm{PF})$, role physical (RP), bodily pain (BP), general health $(\mathrm{GH})$, vitality (VT), social functioning (SF), role emotional $(\mathrm{RE})$, and mental health $(\mathrm{MH})$. The SF-36 also generates a physical component summary (PCS) score, a mental component summary (MCS) score, and a role/social component summary (RCS) score $^{26)}$. The scale scores are calculated by summing responses across scale items and then transforming these raw scores to a $0-100$ scale. Computerized scoring algorithms are available and can be used to produce norm-based $\mathrm{T}$ scores for each scale (with a mean of 50 and SD of 10). Higher scores indicate better health. SF-36 was used under license between our institute and iHope QOL (https://www.sf-36.jp/index.html).

\section{Statistical analysis}

We compared annual survey scores among the following age groups: $20 \mathrm{~s}(n=5), 30 \mathrm{~s}(n=8)$, and $40 \mathrm{~s} / 50 \mathrm{~s}(n=6)$. First, we used the Friedman test to analyze changes in BDI, STAI, EQ-5D, and SF-36 scores over time (Tables 1 and 2). The Wilcoxon signed-rank test and Bonferroni correction were ap-

Table 1. Longitudinal changes in BDI, STAI, and EQ-5D scores during the 4-year period after the disaster

\begin{tabular}{cccccccc}
\hline & Age group & Survey 1 & Survey 2 & Survey 3 & Survey 4 & Survey 5 & Friedman's test \\
\hline \multirow{2}{*}{ BDI } & Total & $10.3(12.5)^{\mathrm{a}}$ & $7.2(6.9)^{\mathrm{b}}$ & $5.3(6.1)$ & $5.7(5.7)$ & $4.2(4.8)^{\mathrm{ab}}$ & $p<0.01$ \\
& $20 \mathrm{~s}$ & $5.0(4.3)$ & $4.8(4.2)$ & $1.2(1.3)$ & $1.8(2.9)$ & $1.4(1.5)$ & 0.01 \\
& $30 \mathrm{~s}$ & $13.9(16.8)$ & $12.4(7.4)^{\mathrm{h}}$ & $9.5(7.3)$ & $6.8(6.5)$ & $6.8(6.1)^{\mathrm{h}}$ & 0.02 \\
& $40 \mathrm{~s} / 50 \mathrm{~s}$ & $9.8(10.3)$ & $2.3(2.3)$ & $3.0(2.5)$ & $7.5(5.5)$ & $3.2(3.0)$ & 0.07 \\
\hline \multirow{2}{*}{ STAI-S } & Total & $51.2(11.1)^{\mathrm{de}}$ & $44.8(8.8)^{\mathrm{c}}$ & $42.2(9.5)$ & $40.9(10.3)^{\mathrm{e}}$ & $34.5(6.9)^{\mathrm{cd}}$ & $p<0.001$ \\
& $20 \mathrm{~s}$ & $46.6(5.9)$ & $46.4(8.4)$ & $34.2(3.9)$ & $39.8(10.3)$ & $31.0(3.8)$ & 0.02 \\
& $30 \mathrm{~s}$ & $49.6(15.1)$ & $47.0(11.1)$ & $49.1(9.7)$ & $38.8(11.2)$ & $37.0(8.8)$ & 0.03 \\
\hline \multirow{2}{*}{ STAI-T } & Total & $47.9(13.1)^{\mathrm{fg}}$ & $39.5(9.6)$ & $40.5(10.7)$ & $37.5(11.6)^{\mathrm{g}}$ & $33.6(9.1)^{\mathrm{f}}$ & $p<0.001$ \\
& $20 \mathrm{~s}$ & $43.4(5.9)$ & $41.0(8.3)$ & $36.4(8.4)$ & $32.2(6.6)$ & $30.8(9.2)$ & 0.03 \\
& $30 \mathrm{~s}$ & $48.6(18.7)$ & $41.9(12.6)$ & $45.4(13.6)$ & $37.4(13.2)$ & $35.8(11.1)$ & 0.10 \\
& $40 \mathrm{~s} / 50 \mathrm{~s}$ & $50.8(8.5)^{\mathrm{jk}}$ & $35.0(4.1)^{\mathrm{k}}$ & $37.3(5.5)$ & $42.2(12.2)$ & $33.2(6.4)^{\mathrm{j}}$ & $p<0.01$ \\
\hline \multirow{2}{*}{ EQ-5D } & Total & $0.914(0.153)$ & $0.928(0.134)$ & $0.960(0.099)$ & $0.972(0.085)$ & $0.946(0.110)$ & 0.03 \\
& $20 \mathrm{~s}$ & $1.000(0.000)$ & $1.000(0.000)$ & $1.000(0.000)$ & $1.000(0.000)$ & $1.000(0.000)$ & 1.00 \\
& $30 \mathrm{~s}$ & $0.901(0.209)$ & $0.886(0.175)$ & $0.958(0.120)$ & $0.961(0.110)$ & $0.934(0.125)$ & 0.16 \\
& $40 \mathrm{~s} / 50 \mathrm{~s}$ & $0.860(0.109)$ & $0.923(0.120)$ & $0.929(0.111)$ & $0.964(0.087)$ & $0.915(0.132)$ & 0.23 \\
\hline
\end{tabular}

Data are shown as mean values (standard deviation).

BDI : Beck depression inventory, STAI : State-Trait Anxiety Inventory, STAI-S : State anxiety scale, STAI-T : Trait anxiety scale, EQ-5D : EuroQoL utility score.

$\mathrm{a}-\mathrm{k}$ : Wilcoxon signed-rank test and Bonferroni correction were performed after Friedman's test.

$\mathrm{a}, \mathrm{b}, \mathrm{e}, \mathrm{g}, \mathrm{h}, \mathrm{k}: p<0.05$

c, d, f, i, j: $p<0.01$ 
Table 2. Longitudinal changes in SF-36 scores during the 4-year period after the disaster

\begin{tabular}{|c|c|c|c|c|c|c|c|}
\hline & Age group & Survey 1 & Survey 2 & Survey 3 & Survey 4 & Survey 5 & $\begin{array}{l}\text { Friedman's } \\
\text { test } p \text {-value }\end{array}$ \\
\hline \multirow[t]{4}{*}{$\mathrm{PF}$} & Total & $54.8(5.4)^{\mathrm{a}}$ & $55.4(4.8)^{\mathrm{b}}$ & $54.6(6.0)$ & $55.8(4.0)^{c}$ & $54.2(6.0)^{\mathrm{abc}}$ & $p<0.001$ \\
\hline & $20 \mathrm{~s}$ & $55.7(3.2)$ & $56.4(3.2)$ & $57.8(0.0)$ & $57.8(0.0)$ & $57.1(1.6)$ & 0.02 \\
\hline & $30 \mathrm{~s}$ & $54.2(7.7)$ & $53.8(6.8)$ & $52.4(8.4)$ & $55.1(3.7)$ & $53.8(7.6)$ & 0.10 \\
\hline & $40 \mathrm{~s} / 50 \mathrm{~s}$ & 54.8 (3.5) & $56.6(1.9)$ & 54.8 (3.5) & $54.8(5.8)$ & $52.4(5.9)$ & 0.08 \\
\hline \multirow[t]{4}{*}{$\mathrm{RP}$} & Total & $50.1(12.3)^{\mathrm{d}}$ & $51.7(13.0)^{f}$ & $54.1(6.9)^{g}$ & $53.1(5.9)^{\mathrm{e}}$ & $52.4(8.2)^{\operatorname{defg}}$ & $p<0.001$ \\
\hline & $20 \mathrm{~s}$ & $49.1(11.5)$ & $55.7(0.0)$ & $55.7(0.0)$ & $55.7(0.0)$ & $55.7(0.0)$ & 0.01 \\
\hline & $30 \mathrm{~s}$ & $49.9(15.2)$ & $46.2(19.4)$ & $52.0(10.6)$ & $51.6(7.9)$ & 50.7 (9.9) & 0.02 \\
\hline & $40 \mathrm{~s} / 50 \mathrm{~s}$ & $51.3(10.9)$ & $55.7(0.0)$ & $55.7(0.0)$ & $53.0(5.3)$ & $51.8(9.5)$ & 0.01 \\
\hline \multirow[t]{4}{*}{$\mathrm{BP}$} & Total & $55.4(9.0)$ & $55.3(10.5)$ & $54.7(7.1)$ & $54.6(8.0)$ & $54.2(7.6)$ & 0.05 \\
\hline & $20 \mathrm{~s}$ & $58.9(3.9)$ & $58.9(3.9)$ & $55.3(6.3)$ & $61.7(0.0)$ & $60.3(3.2)$ & 0.18 \\
\hline & $30 \mathrm{~s}$ & $54.8(10.3)$ & $54.7(15.7)$ & $57.9(5.4)$ & $54.5(9.4)$ & $55.6(5.4)$ & 0.04 \\
\hline & $40 \mathrm{~s} / 50 \mathrm{~s}$ & $53.2(10.5)$ & $53.2(4.8)$ & $50.0(8.2)$ & 48.7 (3.8) & $47.2(7.8)$ & 0.60 \\
\hline \multirow[t]{4}{*}{$\mathrm{GH}$} & Total & $54.9(10.7)$ & $53.8(10.6)$ & $54.5(10.9)$ & $55.5(10.0)$ & $55.4(10.4)$ & 0.79 \\
\hline & $20 \mathrm{~s}$ & $58.4(10.1)$ & $59.1(7.7)$ & $60.0(8.7)$ & $61.8(6.7)$ & $62.1(8.7)$ & 0.49 \\
\hline & $30 \mathrm{~s}$ & $52.4(13.9)$ & $47.6(12.3)$ & $48.6(12.8)$ & $54.2(11.9)$ & $49.9(10.4)$ & 0.14 \\
\hline & $40 \mathrm{~s} / 50 \mathrm{~s}$ & $55.3(5.9)$ & $58.5(3.0)$ & $58.0(6.2)$ & $52.2(8.3)$ & $57.1(8.8)$ & 0.14 \\
\hline \multirow[t]{4}{*}{ VT } & Total & $47.0(11.3)$ & $48.9(8.7)$ & $50.3(8.7)$ & $52.4(8.5)$ & $54.7(8.3)$ & 0.05 \\
\hline & $20 \mathrm{~s}$ & $51.1(7.7)$ & $44.7(5.4)^{\mathrm{r}}$ & $51.8(3.7)$ & $53.7(6.2)$ & $58.2(4.9)^{\mathrm{r}}$ & 0.04 \\
\hline & $30 \mathrm{~s}$ & $45.8(14.0)$ & $48.2(9.6)$ & $45.8(10.5)$ & $54.2(8.7)$ & $50.6(10.7)$ & 0.12 \\
\hline & $40 \mathrm{~s} / 50 \mathrm{~s}$ & $45.0(10.7)$ & $54.3(8.7)$ & $55.2(6.6)$ & $48.8(9.9)$ & $57.4(4.4)$ & 0.02 \\
\hline \multirow[t]{4}{*}{$\mathrm{SF}$} & Total & $44.5(17.5)$ & $52.6(9.6)^{\mathrm{i}}$ & $52.3(11.1)$ & $52.9(10.3)^{\mathrm{h}}$ & $52.6(8.6)^{\mathrm{hi}}$ & $p<0.001$ \\
\hline & $20 \mathrm{~s}$ & $51.9(8.4)$ & $57.0(0.0)$ & $57.0(0.0)$ & $57.0(0.0)$ & $55.7(2.9)$ & 0.01 \\
\hline & $30 \mathrm{~s}$ & $42.5(20.9)$ & $46.5(12.9)$ & $49.0(16.4)$ & $48.2(15.0)$ & $49.0(12.3)$ & 0.22 \\
\hline & $40 \mathrm{~s} / 50 \mathrm{~s}$ & $40.9(19.0)$ & $57.0(0.0)$ & $52.7(5.3)$ & $55.9(2.6)$ & $54.9(3.3)$ & 0.10 \\
\hline \multirow[t]{4}{*}{$\mathrm{RE}$} & Total & $45.1(12.5)^{\mathrm{j}}$ & $52.3(8.3)^{\mathrm{k}}$ & $52.6(10.4)^{1}$ & $52.3(8.0)^{\mathrm{m}}$ & $54.3(5.4)^{\mathrm{jklm}}$ & $p<0.001$ \\
\hline & $20 \mathrm{~s}$ & $48.6(11.2)^{\mathrm{s}}$ & $56.1(0.0)$ & $56.1(0.0)$ & $56.1(0.0)$ & $56.1(0.0)^{\mathrm{s}}$ & $p<0.01$ \\
\hline & $30 \mathrm{~s}$ & $47.7(14.3)^{\mathrm{t}}$ & $47.7(11.6)^{\mathrm{u}}$ & $47.7(15.3)^{\mathrm{v}}$ & $48.8(11.3)$ & $51.9(8.0)^{\mathrm{tuv}}$ & $p<0.01$ \\
\hline & $40 \mathrm{~s} / 50 \mathrm{~s}$ & $38.7(10.3)^{\mathrm{w}}$ & $55.4(1.7)$ & $56.1(0.0)$ & $54.0(3.5)$ & $56.1(0.0)^{\mathrm{w}}$ & $p<0.01$ \\
\hline \multirow[t]{4}{*}{$\mathrm{MH}$} & Total & $45.2(14.4)^{\circ}$ & $49.4(9.2)^{\mathrm{n}}$ & $51.0(9.0)$ & $52.1(8.9)$ & $54.5(7.8)^{\mathrm{no}}$ & $p<0.01$ \\
\hline & $20 \mathrm{~s}$ & $50.2(7.3)$ & $50.7(6.5)$ & $55.6(4.1)$ & $55.6(7.0)$ & $57.7(8.1)$ & 0.06 \\
\hline & $30 \mathrm{~s}$ & $44.8(19.0)$ & $46.1(11.7)$ & $44.8(10.9)$ & $49.8(10.2)$ & $51.2(9.5)$ & 0.13 \\
\hline & $40 \mathrm{~s} / 50 \mathrm{~s}$ & $41.5(12.4)^{\mathrm{x}}$ & $53.4(6.2)$ & $55.4(2.2)$ & $52.3(8.9)$ & $56.3(2.8)^{\mathrm{x}}$ & 0.02 \\
\hline \multirow[t]{4}{*}{ PCS } & Total & $61.4(10.7)^{\mathrm{w}}$ & $56.8(8.2)$ & $56.1(4.9)$ & $56.2(4.8)$ & $53.6(7.6)^{\mathrm{w}}$ & 0.02 \\
\hline & $20 \mathrm{~s}$ & $59.5(8.3)$ & $59.5(4.1)$ & $57.2(5.0)$ & $60.1(2.4)$ & $58.6(7.0)$ & 0.99 \\
\hline & $30 \mathrm{~s}$ & $60.3(8.5)$ & $55.4(11.8)$ & $57.2(4.5)$ & $57.5(4.0)$ & $54.4(5.1)$ & 0.13 \\
\hline & $40 \mathrm{~s} / 50 \mathrm{~s}$ & $64.4(15.5)$ & $56.4(3.9)$ & $53.6(5.3)$ & $51.2(3.1)$ & $48.6(8.8)$ & 0.07 \\
\hline \multirow[t]{4}{*}{ MCS } & Total & $48.3(13.4)$ & $49.7(10.7)$ & $50.5(9.1)$ & $52.4(8.4)$ & $54.6(9.0)$ & 0.15 \\
\hline & $20 \mathrm{~s}$ & $54.2(5.7)$ & $48.9(7.2)$ & $53.8(3.9)$ & $56.3(7.0)$ & $59.3(6.7)$ & 0.17 \\
\hline & $30 \mathrm{~s}$ & $46.3(17.5)$ & $47.7(12.4)$ & $45.7(12.1)$ & $52.6(10.0)$ & $50.3(11.0)$ & 0.10 \\
\hline & $40 \mathrm{~s} / 50 \mathrm{~s}$ & $46.0(12.0)$ & $53.7(6.9)$ & $54.2(3.6)$ & $49.0(6.6)$ & $56.6(5.4)$ & 0.28 \\
\hline \multirow[t]{4}{*}{ RCS } & Total & $40.4(17.2)^{\mathrm{q}}$ & $49.6(10.7)$ & $50.9(10.9)$ & $50.0(10.4)$ & $51.0(8.7)^{\mathrm{q}}$ & 0.02 \\
\hline & $20 \mathrm{~s}$ & $43.4(12.3)$ & $53.7(4.3)$ & $53.5(4.1)$ & $51.1(2.9)$ & $50.3(5.4)$ & 0.05 \\
\hline & $30 \mathrm{~s}$ & $42.3(18.4)$ & 44.7 (14.7) & $47.5(16.3)$ & $45.0(14.0)$ & $49.3(12.1)$ & 0.32 \\
\hline & $40 \mathrm{~s} / 50 \mathrm{~s}$ & $35.4(20.7)$ & $53.4(2.4)$ & $53.3(3.6)$ & $55.7(5.4)$ & $53.9(5.2)$ & 0.33 \\
\hline
\end{tabular}

Data are shown as mean values (standard deviation).

SF-36 : MOS Short-Form 36-item Health Survey, PF : physical functioning, RP : role physical, BP : bodily pain, $\mathrm{GH}$ : general health, VT : vitality, SF : social functioning, RE : role emotional, $\mathrm{MH}$ : mental health, PCS : physical component summary, MCS : mental component summary, RCS : role/social component summary

$\mathrm{a}-\mathrm{x}$ : Wilcoxon signed-rank test and Bonferroni correction were performed after Friedman's test.

a, d, h, o, p, q, r, s, t, u, v, x: $p<0.05$

b, c, e, f, g, i, j, k, l, m, n, w : $p<0.01$ 
plied after the Friedman test to compare data from the different surveys for each age group (Tables 1 and 2). The Mann-Whitney U test and Bonferroni correction were applied after performing the Kruskal-Wallis test to compare scores among the age groups (Tables 3 and 4). A $p$-value of less than 0.05 was considered statistically significant. All analyses were performed using IBM SPSS statistical software (version 26.0, SPSS Inc., Chicago, IL, USA).

\section{Results}

All participants completed the surveys at all annual time points. Although two staff retired from the hospital at age 60 during the survey period, they continued living in Fukushima City, and their questionnaires were collected. None of the participants discontinued working at the Rehabilitation Center because of the Fukushima Daiichi Nuclear Power Plant accident.

\section{$B D I$}

The mean total BDI score at Survey 1 was 10.3 points. The mean score improved with statistical significance over time after Survey 1. Scores for the participants in their 30 s also significantly improved over time (Table 1). However, the scores for participants in their 30s were significantly higher (i.e., worse) than those for participants in their $40 \mathrm{~s} / 50 \mathrm{~s}$ in Survey 2 and those for participants in their 20s in Survey 3 (Table 3, Figure 1).

\section{STAI}

The mean total STAI-S score at Survey 1 was 51.2 points. The score significantly improved over time after Survey 1. The scores for participants in their $40 \mathrm{~s} / 50$ s also significantly improved over time (Table 1). However, the scores for participants in their 30 s were significantly higher (i.e., worse) than those for participants in their 20s in Survey 3 (Table 3 , Figure 2).

The mean total STAI-T score at Survey 1 was

Table 3. Intergenerational changes in BDI, STAI, and EQ-5D scores during the 4-year period after the disaster

\begin{tabular}{lccccc}
\hline & BDI/STAI/EQ-5D & $20 \mathrm{~s}$ & $30 \mathrm{~s}$ & $40 \mathrm{~s} / 50 \mathrm{~s}$ & Kruskal-Wallis test $p$-value \\
\hline Survey 1 & BDI & $5.0(4.3)$ & $13.9(16.8)$ & $9.8(10.3)$ & 0.73 \\
& STAI-S & $46.6(5.9)$ & $49.6(15.1)$ & $57.0(5.9)$ & 0.11 \\
& STAI-T & $43.4(5.9)$ & $48.6(18.7)$ & $50.8(8.5)$ & 0.58 \\
& EQ-5D & $1.000(0.000)$ & $0.901(0.209)$ & $0.860(0.109)$ & 0.11 \\
\hline Survey 2 & BDI & $4.8(4.2)$ & $12.4(7.4)^{\mathrm{a}}$ & $2.3(2.3)^{\mathrm{a}}$ & 0.01 \\
& STAI-S & $46.4(8.4)$ & $47.0(11.1)$ & $40.5(4.0)$ & 0.46 \\
& STAI-T & $41.0(8.3)$ & $41.9(12.6)$ & $35.0(4.1)$ & 0.40 \\
& EQ-5D & $1.000(0.000)$ & $0.886(0.175)$ & $0.923(0.120)$ & 0.32 \\
\hline \multirow{2}{*}{ Survey 3 } & BDI & $1.2(1.3)^{\mathrm{b}}$ & $9.5(7.3)^{\mathrm{b}}$ & $3.0(2.5)$ & 0.01 \\
& STAI-S & $34.2(3.9)^{\mathrm{c}}$ & $49.1(9.7)^{\mathrm{c}}$ & $39.7(5.8)$ & 0.04 \\
& STAI-T & $36.4(8.4)$ & $45.4(13.6)$ & $37.3(5.5)$ & 0.22 \\
& EQ-5D & $1.000(0.000)$ & $0.958(0.120)$ & $0.929(0.111)$ & 0.38 \\
& BDI & $1.8(2.9)$ & $6.8(6.5)$ & $7.5(5.5)$ & 0.17 \\
& STAI-S & $39.8(10.3)$ & $38.8(11.2)$ & $44.7(9.6)$ & 0.62 \\
& STAI-T & $32.2(6.6)$ & $37.4(13.2)$ & $42.2(12.2)$ & 0.20 \\
& EQ-5D & $1.000(0.000)$ & $0.961(0.110)$ & $0.964(0.087)$ & 0.68 \\
\hline \multirow{2}{*}{ Survey 5 } & BDI & $1.4(1.5)$ & $6.8(6.1)$ & $3.2(3.0)$ & 0.16 \\
& STAI-S & $31.0(3.8)$ & $37.0(8.8)$ & $34.2(5.5)$ & 0.27 \\
& STAI-T & $30.8(9.2)$ & $35.8(11.1)$ & $33.2(6.4)$ & 0.67 \\
& EQ-5D & $1.000(0.000)$ & $0.934(0.125)$ & $0.915(0.132)$ & \\
\hline
\end{tabular}

Data are shown as mean values (standard deviation).

BDI : Beck depression inventory, STAI : State-Trait Anxiety Inventory, STAI-S : State anxiety scale, STAI-T : Trait anxiety scale, EQ-5D : EuroQoL utility score.

a, b, c : Mann-Whitney U test and Bonferroni correction were performed after Kruskal-Wallis test.

a, b, c : $p<0.05$ 
Table 4. Intergenerational changes in SF-36 scores during the 4-year period after the disaster

\begin{tabular}{|c|c|c|c|c|c|}
\hline & SF-36 & $20 \mathrm{~s}$ & $30 \mathrm{~s}$ & $40 \mathrm{~s} / 50 \mathrm{~s}$ & Kruskal-Wallis test $p$-value \\
\hline \multirow[t]{11}{*}{ Survey 1} & $\mathrm{PF}$ & $55.7(3.2)$ & $54.2(7.7)$ & $54.8(3.5)$ & 0.81 \\
\hline & $\mathrm{RP}$ & $49.1(11.5)$ & $49.9(15.2)$ & $51.3(10.9)$ & 0.74 \\
\hline & $\mathrm{BP}$ & $58.9(3.9)$ & $54.8(10.3)$ & $53.2(10.5)$ & 0.70 \\
\hline & $\mathrm{GH}$ & $58.4(10.1)$ & $52.4(13.9)$ & $55.3(5.9)$ & 0.62 \\
\hline & VT & $51.1(7.7)$ & $45.8(14.0)$ & $45.0(10.7)$ & 0.56 \\
\hline & $\mathrm{SF}$ & $51.9(8.4)$ & $42.5(20.9)$ & $40.9(19.0)$ & 0.73 \\
\hline & $\mathrm{RE}$ & $48.6(11.2)$ & $47.7(14.3)$ & $38.7(10.3)$ & 0.19 \\
\hline & $\mathrm{MH}$ & $50.2(7.3)$ & $44.8(19.0)$ & $41.5(12.4)$ & 0.54 \\
\hline & PCS & $59.5(8.3)$ & $60.3(8.5)$ & $64.4(15.5)$ & 0.86 \\
\hline & MCS & $54.2(5.7)$ & $46.3(17.5)$ & $46.0(12.0)$ & 0.63 \\
\hline & RCS & $43.4(12.3)$ & $42.3(18.4)$ & $35.4(20.7)$ & 0.68 \\
\hline \multirow[t]{11}{*}{ Survey 2} & $\mathrm{PF}$ & $56.4(6.2)$ & $53.8(6.8)$ & $56.6(1.9)$ & 0.76 \\
\hline & $\mathrm{RP}$ & $55.7(0.0)$ & $46.2(19.4)$ & $55.7(0.0)$ & 0.23 \\
\hline & $\mathrm{BP}$ & $58.9(3.9)$ & 54.7 (15.7) & $53.2(4.8)$ & 0.13 \\
\hline & GH & $59.1(7.7)$ & $47.6(12.3)$ & $58.5(3.0)$ & 0.09 \\
\hline & VT & $44.7(5.4)$ & $48.2(9.6)$ & $54.3(8.7)$ & 0.21 \\
\hline & $\mathrm{SF}$ & $57.0(0.0)$ & $46.5(12.9)$ & $57.0(0.0)$ & 0.04 \\
\hline & $\mathrm{RE}$ & $56.1(0.0)$ & 47.7 (11.6) & $55.4(1.7)$ & 0.23 \\
\hline & $\mathrm{MH}$ & 50.7 (6.5) & $46.1(11.7)$ & $53.4(6.2)$ & 0.40 \\
\hline & PCS & 59.5 (4.1) & $55.4(11.8)$ & $56.4(3.9)$ & 0.62 \\
\hline & MCS & $48.9(7.2)$ & $47.7(12.4)$ & $53.7(6.9)$ & 0.47 \\
\hline & RCS & $53.7(4.3)$ & 44.7 (14.7) & $53.4(2.4)$ & 0.27 \\
\hline \multirow[t]{11}{*}{ Survey 3} & $\mathrm{PF}$ & $57.8(0.0)$ & $52.4(8.4)$ & $54.8(3.5)$ & 0.08 \\
\hline & $\mathrm{RP}$ & $55.7(0.0)$ & $52.0(10.6)$ & $55.7(0.0)$ & 0.50 \\
\hline & $\mathrm{BP}$ & $55.3(6.3)$ & $57.9(5.4)$ & $50.0(8.2)$ & 0.14 \\
\hline & $\mathrm{GH}$ & $60.0(8.7)$ & $48.6(12.8)$ & $58.0(6.2)$ & 0.22 \\
\hline & VT & $51.8(3.7)$ & 45.8 (10.5) & $55.2(6.6)$ & 0.25 \\
\hline & SF & $57.0(0.0)$ & $49.0(16.4)$ & 52.7 (5.3) & 0.27 \\
\hline & $\mathrm{RE}$ & $56.1(0.0)$ & 47.7 (15.3) & $56.1(0.0)$ & 0.10 \\
\hline & $\mathrm{MH}$ & $55.6(4.1)$ & 44.8 (10.9) & $55.4(2.2)$ & 0.08 \\
\hline & PCS & $57.2(5.0)$ & $57.2(4.5)$ & $53.6(5.3)$ & 0.33 \\
\hline & MCS & $53.8(3.9)$ & 45.7 (12.1) & $54.2(3.6)$ & 0.24 \\
\hline & RCS & $53.5(4.1)$ & 47.5 (16.3) & $53.3(3.6)$ & 0.96 \\
\hline \multirow[t]{11}{*}{ Survey 4} & $\mathrm{PF}$ & $57.8(0.0)$ & $55.1(3.7)$ & $54.8(5.8)$ & 0.21 \\
\hline & $\mathrm{RP}$ & $55.7(0.0)$ & $51.6(7.9)$ & $53.0(5.3)$ & 0.42 \\
\hline & $\mathrm{BP}$ & $61.7(0.0)^{\mathrm{a}}$ & $54.5(9.4)$ & $48.7(3.8)^{\mathrm{a}}$ & 0.01 \\
\hline & $\mathrm{GH}$ & $61.8(6.7)$ & $54.2(11.9)$ & $52.2(8.3)$ & 0.25 \\
\hline & VT & $53.7(6.2)$ & $54.2(8.7)$ & 48.8 (9.9) & 0.72 \\
\hline & $\mathrm{SF}$ & $57.0(0.0)$ & $48.2(15.0)$ & $55.9(2.6)$ & 0.25 \\
\hline & $\mathrm{RE}$ & $56.1(0.0)$ & 48.8 (11.3) & $54.0(3.5)$ & 0.29 \\
\hline & $\mathrm{MH}$ & $55.6(7.0)$ & $49.8(10.2)$ & $52.3(8.9)$ & 0.60 \\
\hline & PCS & $60.1(2.4)^{\mathrm{b}}$ & $57.5(4.0)$ & $51.2(30.1)^{\mathrm{b}}$ & 0.01 \\
\hline & MCS & $56.3(7.0)$ & $52.6(10.0)$ & $49.0(6.6)$ & 0.22 \\
\hline & RCS & $51.1(2.9)$ & $45.0(14.0)$ & 55.7 (5.4) & 0.22 \\
\hline \multirow[t]{11}{*}{ Survey 5} & $\mathrm{PF}$ & $57.1(1.6)$ & $53.8(7.6)$ & $52.4(5.9)$ & 0.28 \\
\hline & $\mathrm{RP}$ & $55.7(0.0)$ & 50.7 (9.9) & $51.8(9.5)$ & 0.51 \\
\hline & $\mathrm{BP}$ & $60.3(3.2)^{c}$ & $55.6(5.4)$ & $47.2(7.8)^{c}$ & 0.01 \\
\hline & $\mathrm{GH}$ & $62.1(8.7)$ & $49.9(10.4)$ & $57.1(8.8)$ & 0.19 \\
\hline & VT & $58.2(4.9)$ & $50.6(10.7)$ & $57.4(4.4)$ & 0.23 \\
\hline & SF & $55.7(2.9)$ & $49.0(12.3)$ & 54.9 (3.3) & 0.62 \\
\hline & $\mathrm{RE}$ & $56.1(0.0)$ & $51.9(8.0)$ & $56.1(0.0)$ & 0.23 \\
\hline & $\mathrm{MH}$ & $57.7(8.1)$ & $51.2(9.5)$ & $56.3(2.8)$ & 0.30 \\
\hline & PCS & $58.6(7.0)$ & $54.4(5.1)$ & $48.6(8.8)$ & 0.07 \\
\hline & MCS & $59.3(6.7)$ & $50.3(11.0)$ & $56.6(5.4)$ & 0.12 \\
\hline & RCS & $50.3(5.4)$ & $49.3(12.1)$ & $53.9(5.2)$ & 0.66 \\
\hline
\end{tabular}

Data are shown as mean values (standard deviation).

SF-36 : MOS Short-Form 36-item Health Survey, PF : physical functioning, RP : role physical, BP : bodily pain, GH : general health, VT : vitality, SF : social functioning, RE : role emotional, MH : mental health, PCS : physical component summary, MCS : mental component summary, RCS : role/social component summary

a, b, c : Mann-Whitney U test and Bonferroni correction were performed after Kruskal-Wallis test.

a, b, c : $p<0.01$ 


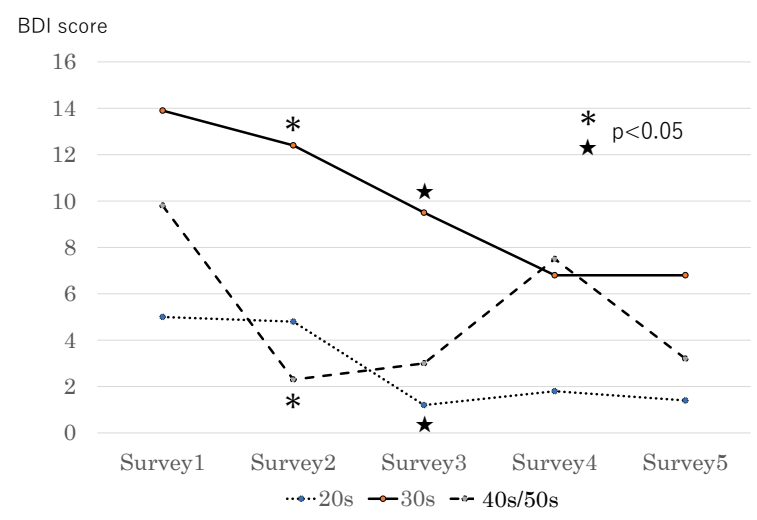

Fig. 1. Longitudinal changes in BDI score

The scores for participants in their 30s were significantly higher (i.e., worse) than those for participants in their $40 \mathrm{~s} / 50 \mathrm{~s}$ in Survey 2 and those for participants in their 20s in Survey 3.

We applied a Mann-Whitney U test and Bonferroni correction after conducting a Kruskal-Wallis test. BDI : Beck Depression Inventory

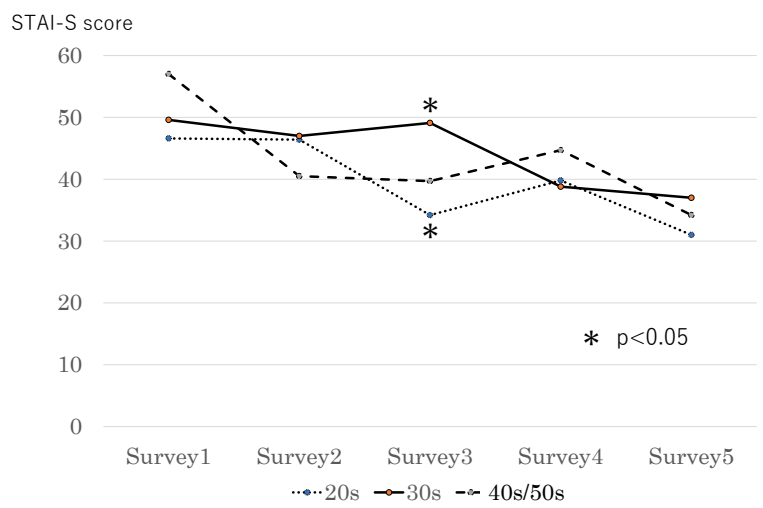

Fig. 2. Longitudinal changes in STAI-S scores

The scores for participants in their 30s were significantly higher (i.e., worse) than those for participants in their 20s in Survey 3.

We applied a Mann-Whitney U test and Bonferroni correction after conducting a Kruskal-Wallis test.

STAI-S : State-Trait Anxiety Inventory-State

47.9 points. This score significantly improved over time after Survey 1. The score for participants in their $40 \mathrm{~s} / 50 \mathrm{~s}$ also significantly improved over time (Table 1). There were no significant differences between the age groups (Table 3, Figure 3 ).

\section{$E Q-5 D$}

The mean EQ-5D score in Survey 1 was 0.914 . This score was stable and did not significantly change with time (Table 1). Additionally, there were no significant differences among age groups (Table 3, Figure 4).

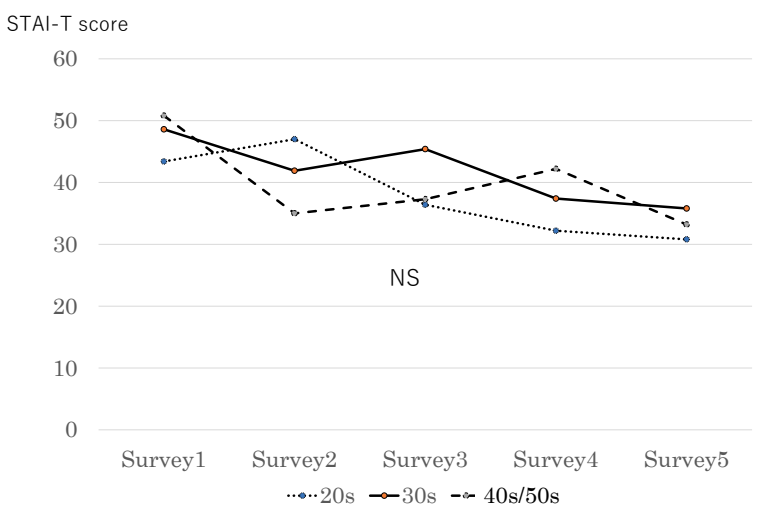

Fig. 3 Longitudinal changes in STAI-T scores There were no significant differences among the age groups.

We applied a Mann-Whitney U test and Bonferroni correction after conducting a Kruskal-Wallis test. STAI-T : State-Trait Anxiety Inventory-Trait

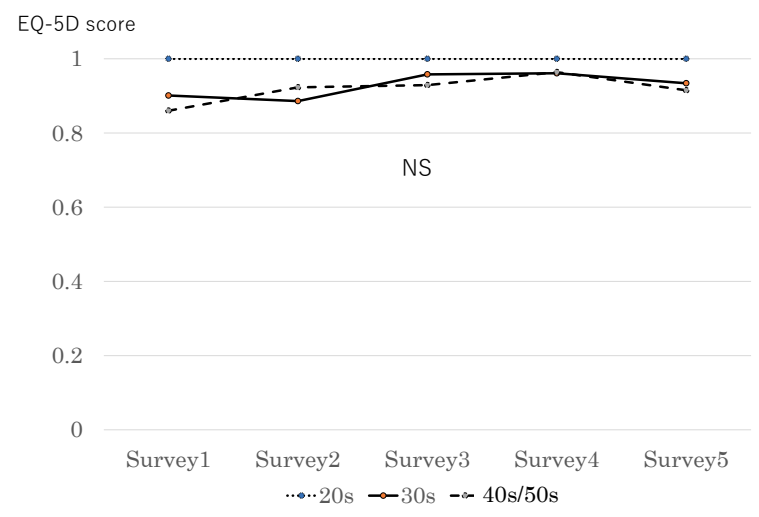

Fig. 4 Longitudinal changes in EQ-5D scores There were no significant differences among the age groups.

We applied a Mann-Whitney U test and Bonferroni correction after conducting a Kruskal-Wallis test. EQ-5D : EuroQol-5 dimensions

\section{$S F-36$}

The total subscale scores for PF, RP, SF, RE, and $\mathrm{MH}$ significantly improved over time after Survey 1 . VT scores among participants in their 20 s and RE scores among those in their 20s, 30s, and 40s/50s significantly improved over time. Additionally, PCS and RCS scores significantly improved over time (Table 2).

A comparison between the age groups showed that BP scores among participants in their $40 \mathrm{~s} / 50 \mathrm{~s}$ were significantly lower (i.e., worse) than those among participants in their 20s in Survey 4 and those in their 30s in Survey 5 (Table 4, Figure 5). The Survey 4 PCS scores were also significantly lower among participants in their $40 \mathrm{~s} / 50$ s than 
among participants in their 20s (Table 4, Figure 6).

\section{Discussion}

This longitudinal study on psychologic distress and QOL in medical staff after a disaster demonstrated a tendency toward recovery. However, there were differences among age groups in some longitudinal changes, such as the recovery period.

\section{Psychologic distress}

Disasters, such as pandemics or nuclear power plant accidents, are likely to affect mental health in

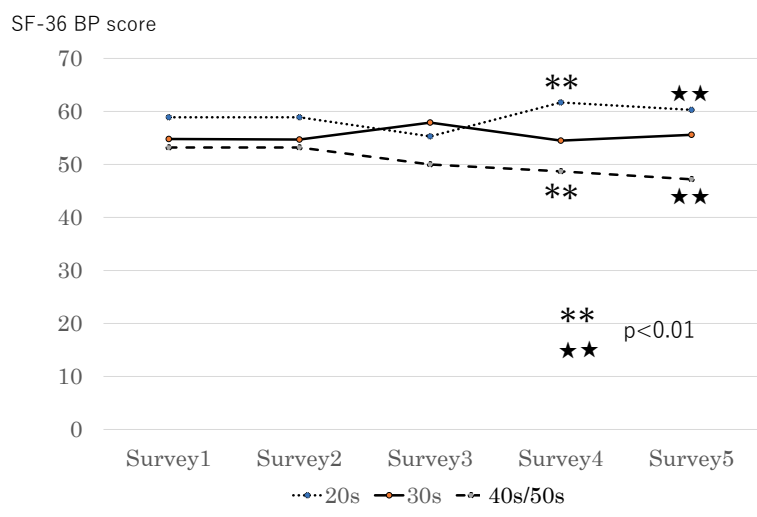

Fig. 5 Longitudinal changes in SF-36 BP scores BP scores for participants in their $40 \mathrm{~s} / 50$ s were significantly lower (i.e., worse) than those for participants in their 20s in Survey 4 and Survey 5. We applied a Mann-Whitney U test and Bonferroni correction after conducting a Kruskal-Wallis test. SF-36 : MOS Short-Form 36-item Health Survey BP : bodily pain

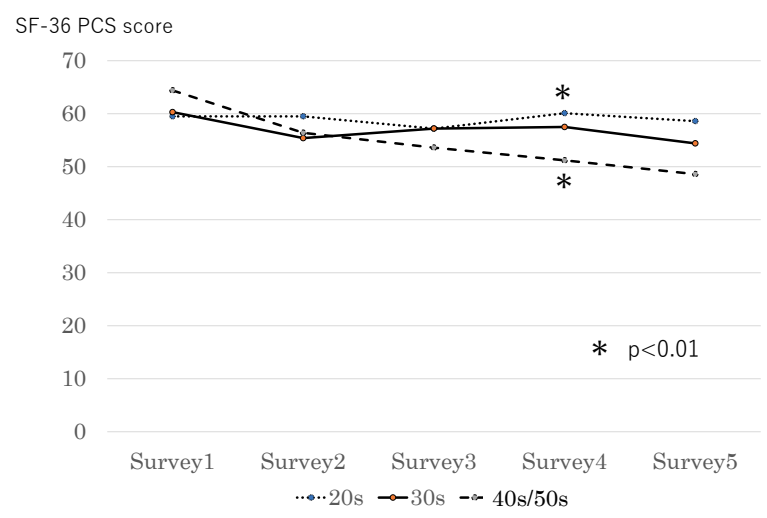

Fig. 6. Longitudinal changes in SF-36 PCS scores PCS scores in Survey 4 were significantly lower among participants in their $40 \mathrm{~s} / 50$ s compared with participants in their 20 s.

We applied a Mann-Whitney U test and Bonferroni correction after conducting a Kruskal-Wallis test. SF-36 : MOS Short-Form 36-item Health Survey PCS : physical component summary affected populations in general and medical staff in particular. Several studies have assessed mental health in public health nurses ${ }^{27)}$ and disaster medical assistance team members ${ }^{28,29)}$. However, these cross-sectional studies did not examine changes in mental health over time.

Several longitudinal studies have examined changes in mental health in caregivers and residents in Fukushima Prefecture. Fujitani et al. reported that a substantial number of caregivers working in Fukushima Prefecture exhibited signs of emotional exhaustion, feelings of low personal accomplishment, and psychologic distress 2 years after the Fukushima Daiichi Nuclear Power Plant disas$\operatorname{ter}^{10)}$. Oe et al. reported that 3 years after the Fukushima Daiichi Nuclear Power Plant accident, the prevalence of general psychologic distress among residents in the evacuation zone was higher in both men and women, compared with normal Japanese levels in non-disaster settings ${ }^{11)}$. In addition, Orui et al. reported that evacuees and residents from the area affected by the Fukushima Daiichi Nuclear Power Plant accident exhibited signs of delayed mental health recovery 7 years after the disaster $^{12)}$. These results suggest that disaster-related negative effects on mental health can persist for several years or more. Our results for medical staff indicate that disaster-related depression and anxiety significantly improved over time. However, recovery was delayed among participants in their $30 \mathrm{~s}$ compared with participants in their $20 \mathrm{~s}$ and $40 \mathrm{~s} / 50 \mathrm{~s}$. This may reflect the likelihood that those in their 30 s have young families that either evacuated while medical workers remained, or, if families stayed together, there may have been greater concerns about exposure to low-dose radiation. BDI and STAI scores at 3 years (Survey 4) and 4 years (Survey 5) after the disaster were not significantly different. Thus, follow-up assessments of psychologic distress in medical staff should be continued for at least 3 years, especially for participants in their 30s. Risk factors associated with distress measures during the COVID-19 pandemic include female gender, younger age ( $\leq 40$ years), and frequent exposure to social media/news about COVID-19 ${ }^{30)}$.

Kohzaki et al. administered a questionnaire survey to citizens, doctors, and medical students both inside and outside Fukushima in 2011 and 2013 ${ }^{31}$. They reported that medical students who had recently studied radiation biology showed much less ongoing anxiety compared with other groups. This suggests that appropriate knowledge of the risks associated with a disaster can help to reduce anxiety. 


\section{QOL}

QOL, as evaluated by the EQ-5D, was relatively high 12 days after the disaster (Survey 1) and did not change over the following 4 years. However, scores on five of the eight SF-36 subscales, as well as the RCS score, significantly improved over time. In contrast, the PCS score significantly decreased over time. Participants in their $40 \mathrm{~s} / 50 \mathrm{~s}$ obtained significantly lower BP and PCS scores compared with participants in their 20s. These findings suggest that the SF-36 is able to evaluate health-related QOL in greater detail than the EQ5D. Furthermore, among participants in their $40 \mathrm{~s} / 50 \mathrm{~s}$, physical QOL decreased over time, although mental QOL significantly improved. However, this decline in physical-health QOL among participants in their $40 \mathrm{~s} / 50$ s may have more to do with physiological changes related to aging ${ }^{32)}$. Khachadourian et al. examined the associations between demographic characteristics, trauma exposure, and psychosocial variables in terms of QOL 23 years after the 1988 Spitak earthquake in Armenia $^{33)}$. They found that older age, current depression, post-traumatic stress disorder, and anxiety were negatively associated with QOL. These data indicate that age is an important factor in disaster responses, and that long-term follow-up assessments of QOL should be conducted in affected individuals.

There are several limitations to the present study. First, the number of participants was small, and came from a single institution's rehabilitation center. Second, the follow-up period was not particularly long for evaluating post-disaster changes in mental health and QOL. Third, the versions of the questionnaires used in this study were not the most recent. The $\mathrm{EQ}-5 \mathrm{D}$ has been updated to the $\mathrm{EQ}^{-}$ $5 \mathrm{D}-5 \mathrm{~L}^{34)}$ and the BDI to the BDI-II ${ }^{15)}$. Fourth, this study did not include medical staff working directly in disaster response or radiation emergency medicine, who may have experienced different psychologic trajectories. Fifth, we did not include a control group. To demonstrate more conclusively that the disaster had a direct effect on participants, a comparison group that was not exposed to the disaster would be needed. Finally, in this study, we did not evaluate participants' families. Psychologic distress experienced by family members may affect the mental health of participants. Further studies that include family members are needed to more accurately evaluate the effect of disasters on medical staff.
The authors believe that the strengths of the study are the following. First, the survey involved multiple assessment tools. Second, it was rapidly implemented at a hospital directly affected by the Great East Japan Earthquake and directly involved in responding to the combined earthquake, tsunami, and nuclear crisis. Third, although limited to a single center at a single institution, a conscientious informed consent process secured 100\% participation across the full spectrum of professions employed by the center. Fourth, voluntary participation was sustained throughout 5 annual surveys, even among staff who retired during the observation period.

In conclusion, a compound disaster can affect levels of psychologic distress and QOL among medical staff, including those not directly involved in disaster response. The finding that longitudinal changes such as recovery period differed among age groups leads us to believe that age is an important consideration in the pathology of psychologic distress and in the pathway to improving QOL in medical staff after a disaster. We dare to imagine that our study will inspire readers to anticipate the need for similar post-disaster investigations, in order proceed with similar speed, larger cohorts, and appropriate control groups.

\section{Acknowledgment}

We would like to express our gratitude to the staff working at the Rehabilitation Center, Fukushima Medical University Hospital, for continuing to work after the disaster and for completing the questionnaire five times over a 4-year period. We are also grateful to Associate Professor Kyoko Shibata, $\mathrm{PhD}$, for making useful comments regarding the statistical analysis. We thank Sydney Koke, MFA, and Diane Williams, PhD, from Edanz Group (https:// jp.edanz.com/ac) for editing a draft of this manuscript.

\section{Conflict of interest disclosure}

We have no conflicts of interest pertaining to this manuscript.

\section{References}

1. Gao J, Zheng P, Jia Y, et al. Mental health problems and social media exposure during COVID-19 outbreak. PLoS One, 15 : e0231924, 2020.

2. Rogers JP, Chesney E, Oliver D, et al. Psychiatric and neuropsychiatric presentations associated with 
severe coronavirus infections: A systematic review and meta-analysis with comparison to the COVID-19 pandemic. Lancet Psychiat, 7 : 611627, 2020.

3. Chen Q, Liang M, Li Y, et al. Mental health care for medical staff in China during the COVID-19 outbreak. Lancet Psychiat, 7 : e15-16, 2020.

4. Huang J, Liu F, Teng Z, et al. Care for the psychological status of frontline medical staff fighting against COVID-19. Clin Infect Dis, Apr 3 : ciaa385, 2020.

5. Ripp J, Peccoralo L, Charney D. Attending to the emotional well-being of the health care workforce in a New York City health system during the COVID-19 pandemic. Acad Med, 95 : 1136-1139, 2020.

6. Labrague LJ, de Los Santos JAA. Fear of COVID-19, psychological distress, work satisfaction and turnover intention among frontline nurses. J Nurs Manag, 29 : 395-403, 2021.

7. Yabe H, Suzuki Y, Mashiko H, et al. Psychological distress after the Great East Japan Earthquake and Fukushima Daiichi Nuclear Power Plant accident : Results of a mental health and lifestyle survey through the Fukushima Health Management Survey in FY2011 and FY2012. Fukushima J Med Sci, 60 : 57-67, 2014.

8. Harada N, Shigemura J, Tanichi M, Kawaida K, Takahashi S, Yasukata F. Mental health and psychological impacts from the 2011 Great East Japan Earthquake disaster: A systematic literature review. Disaster Mil Med, Sep 2 : 1-17, 2015.

9. Yabuki S, Ouchi K, Kikuchi S, Konno S. Pain, quality of life and activity in aged evacuees living in temporary housing after the Great East Japan Earthquake of 11 March 2011: A cross-sectional study in Minamisoma City, Fukushima Prefecture. BMC Musculoskelet Disord, 16:246, 2015.

10. Fujitani K, Carroll M, Yanagisawa R, Katz C. Burnout and psychiatric distress in local caregivers two years after the 2011 Great East Japan Earthquake and Fukushima Nuclear Radiation disaster. Community Ment Health J, 52 : 39-45, 2016.

11. Oe M, Fujii S, Meda M, et al. Three-year trend survey of psychological distress, post-traumatic stress, and problem drinking among residents in the evacuation zone after the Fukushima Daiichi Nuclear Power Plant accident [The Fukushima Health Management Survey]. Psychiatry Clin Neurosci, 70 : 245-252, 2016.

12. Orui M, Nakajima S, Takebayashi Y, et al. Mental health recovery of evacuees and residents from Fukushima Daiichi Nuclear Power Plant accident after seven years-Contribution of social network and a desirable lifestyle. Int J Environ Res Public
Health, 15 : 2381, 2018.

13. Nishi D, Susukida R, Usuda K, Yamanouchi Y. Psychological distress among people in Fukushima prefecture before and after the Great East Japan Earthquake using a nation-wide survey. Psychiatry Clin Neurosci, 72 : 876-878, 2018.

14. Murakami M, Kobayashi T, Ochi S, Goto A, Igarashi Y. Thinking about COVID-19 from Fukushima and Fukushima from COVID-19. (in Japanese) Japanese Journal of Risk Analysis, 30 : 195-202, 2021.

15. Smarr KL, Keefer AL. Measures of depression and depressive symptoms. Arthrit Care Res, 63 : S454-466, 2011.

16. Beck AT, Steer RA. Manual for the Beck Depression Inventory. The Psychological Corporation, San Antonio (TX), 1987.

17. Spielberger CD, Gorsuch RL, Lushene RE. STAI Manual. Consulting Psychologists Press, California, 1970.

18. Julian LJ. Measures of anxiety. Arthrit Care Res, 63 : S467-472, 2011.

19. Addolorato G, Ancona C, Capristo E, et al. State and trait anxiety in women affected by allergic and vasomotor rhinitis. J Psychosom Res, 46 : 283289, 1999.

20. Tsuchiya A, Ikeda S, Ikegami N, et al. Estimating an EQ-5D population value set: The case of Japan. Health Econ, 11 : 341-353, 2002.

21. Brooks R with the EuroQol Group. EuroQol : The current state of play. Health Policy, 37 : 5372, 1996.

22. EuroQol Group. EuroQol-A new facility for the measurement of health-related quality of life. Health Policy, 16 : 199-208, 1990.

23. Fukuhara S, Ware JE Jr, Kosinski M, Wada S, Gandek B. Psychometric and clinical tests of validity of the Japanese SF-36 Health Survey. J Clin Epidemiol, 51 : 1045-1053, 1998.

24. Fukuhara S, Bito S, Green J, Hsiao A, Kurokawa K. Translation, adaptation, and validation of the SF-36 Health Survey for use in Japan. J Clin Epidemiol, 51 : 1037-1044, 1998.

25. Busija L, Pausenberger E, Haines TP, Haymes S, Buchbinder R, Osborne RH. Adult measures of general health and health-related quality of life. Arthrit Care Res, 63 : S383-412, 2011.

26. Suzukamo Y, Fukuhara S, Green J, Kosinski M, Gandek B, Ware JE. Validation testing of a threecomponent model of Short Form-36 scores. J Clin Epidemiol, 64 : 301-308, 2011.

27. Yokoyama Y, Hirano K, Sato M, et al. Activities and health status of dispatched public health nurses after the great East Japan earthquake. Public Health Nurs, 31 : 537-544, 2014.

28. Matsuoka Y, Nishi D, Nakaya N, et al. Concern 
over radiation exposure and psychological distress among rescue workers following the Great East Japan Earthquake. BMC Public Health, 12 : 249, 2012.

29. Nishi D, Koido Y, Nakaya N, et al. Peritraumatic distress, watching television, and posttraumatic stress symptoms among rescue workers after the Great East Japan Earthquake. PLoS One, 7 : e35248, 2012.

30. Xiong J, Lipsitz O, Nasri F, et al. Impact of COVID-19 pandemic on mental health in the general population : A systematic review. J Affect Disord, 277 : 55-64, 2020.

31. Kohzaki M, Ootsuyama A, Moritake T, Abe T, Kubo T, Okazaki R. What have we learned from a ques- tionnaire survey of citizens and doctors both inside and outside Fukushima? : Survey comparison between 2011 and 2013. J Radiol Prot, 35 : N1-17, 2015.

32. Tieland M, Trouwborst I, Clark BC. Skeletal muscle performance and aging. J Cachexia Sarcopenia Muscle, 9 : 3-19, 2018.

33. Khachadourian V, Armenian HK, Demirchyan A, Goenjian A. Loss and psychosocial factors as determinants of quality of life in a cohort of earthquake survivors. Health Qual Life Outcomes, 13 : 13, 2015.

34. Devlin NJ, Brooks R. EQ-5D and the EuroQol group : Past, present and future. Appl Health Econ Health Policy, 15 : 127-137, 2011. 